\title{
Évaluation du raisonnement analogique et des connaissances relationnelles d'enfants présentant une dysphasie.
}

\section{Evaluation of analogical reasoning and relational knowledge of children with Specific}

\section{Language Impairment.}

\author{
Krzemien Magali, Leroy Sandrine, Maillart Christelle \\ Département de Psychologie : Cognition et Comportement, Université de Liège, Liège, Belgique \\ Correspondance: Magali Krzemien, B.38, 30 rue de l'Aunaie, 4000 Liège, 04/366.59.89, \\ magali.krzemien@gmail.com
}

Certains auteurs soutiennent que les difficultés langagières des enfants présentant une dysphasie seraient liées à leurs faibles capacités de raisonnement analogique par rapport à leurs pairs de même âge [20]. Nous cherchons donc ici à préciser la nature de ces difficultés. Une tâche de scènes analogiques a été proposée à un groupe d'enfants présentant une dysphasie et à des groupes contrôles appariés en âge chronologique, en âge linguistique et en mémoire de travail. La nature des indices perceptuels présents dans chaque item variait selon la condition. Ainsi, tous les enfants semblent bénéficier de la congruence entre informations perceptuelles et relationnelles. De plus, les enfants présentant une dysphasie semblent posséder des connaissances relationnelles relativement préservées par rapport à leurs pairs de même âge, mais des difficultés apparaissent quand la tâche se complexifie, notamment quand des distracteurs perceptuels doivent être inhibés. Or, aucune différence n'apparait entre les enfants présentant une dysphasie et leurs pairs de même âge linguistique ou de capacités de mémoire de travail similaires. Leurs difficultés pourraient donc être expliquées par les troubles langagiers et les déficits en mémoire de travail liés à la dysphasie.

Analogical reasoning is a human ability that allows making a correspondence between two situations based on their common relational structure. It permits to learn new information about a specific situation and it is also strongly linked to language abilities. Thus, according to some authors [20], the language disorders of children with Specific Language Impairment (SLI) are due to analogical reasoning difficulties. These children seem indeed to have problems to abstract a structure from several heard forms. In this paper, we will try to understand more accurately these analogical reasoning difficulties. To reach this goal, we created a scene analogy task that was administered to a group of children with SLI. The children were matched to a chronological age, a linguistic age and a working memory control participant. Perceptual infomation wich varied accross conditions was added. The perceptual similarities were in agreement with the relational similarities or not, or were absent from the items. It seems that all children benefit from the agreement between perceptual and relational information. Children with SLI also seem to have similar relational knowledge than their chronological age peers. However, they tend to have difficulties applying an analogical reasoning when perceptual information has to be inhibited. These difficulties could be explained by their poor language and working memory abilities since no difference appears between children with SLI and their linguistic age or working memory peers. Yet, a qualitative impairment of relational knowledge cannot be excluded. 
Mots-clés/Keywords : Développement du langage/Language Development, Troubles du développement du langage/Language Development Disorders, Mémoire de travail/Working Memory.

\section{INTRODUCTION}

Le raisonnement analogique désigne la capacité à établir une correspondance entre deux situations : une situation de base et une situation cible [1]. Il nécessite de découvrir la structure commune entre deux situations et de pouvoir faire correspondre un ensemble de relations à un autre [2]. Le fait d'utiliser de telles analogies permet de comprendre le domaine cible et de dresser des inférences quant à ses propriétés ou son fonctionnement. Ainsi, si l'on tente d'expliquer le métabolisme d'une cellule par une analogie utilisant le feu, on peut mettre en correspondance différents éléments comme le fait que le feu et la cellule produisent tous deux de l'énergie grâce à l'oxygène. En sachant que le feu libère de l'eau et du dioxyde de carbone, on peut également inférer qu'une cellule en libère aussi [1]. Le raisonnement analogique permet donc d'apprendre de nouvelles connaissances à partir de situations familières et connues [3].

\section{Raisonnement analogique, connaissance relationnelle et mémoire de travail}

Selon certains modèles, l'émergence du raisonnement analogique relèverait d'un stade de développement particulier atteint à l'adolescence. D'autres modèles soulignent l'importance des connaissances relationnelles détenues par l'enfant pour raisonner analogiquement. Ainsi, Gentner [4] soutient que le développement du raisonnement analogique dépend d'une transition entre la prise en compte du perceptuel et celle du relationnel. En effet, selon elle, les enfants progressent d'une aptitude à raisonner sur les caractéristiques physiques des objets à une aptitude à raisonner à partir de leurs relations, ce qui définit le raisonnement analogique. Cette évolution pourrait être expliquée par l'accroissement des connaissances relatives à un domaine spécifique avec l'âge. L'échec à une tâche refléterait en fait le manque de connaissances relatives à celle-ci, connaissances qui sont nécessaires à la prise en compte des éléments relationnels par rapport aux perceptuels. Selon cette hypothèse, la transition du perceptuel au relationnel serait donc spécifique à chaque domaine et pourrait survenir à différents moments selon les connaissances accumulées [4].

Goswami [2] souligne également le rôle primordial des connaissances des relations présentes au sein d'une tâche analogique. Elle soutient en effet que les enfants sont capables 
de raisonner analogiquement dès leur plus jeune âge, pour peu qu'ils détiennent une connaissance suffisante des relations en présence. Ainsi, Goswami et Brown [5] obtiennent des taux de réussite importants dans une tâche d'analogie basée sur des relations causales chez des enfants de 3, 4 et 6 ans. Contrairement à d'autres études, les auteurs se sont assurés ici que les enfants connaissaient les relations en présence. Ainsi, on retrouvait des transformations de type «pâte à modeler : pâte à modeler coupée » puis «pomme : pomme coupée ». Selon Goswami et Brown [5], il est donc primordial d'utiliser des relations que les enfants comprennent pour qu'ils puissent raisonner analogiquement.

Les connaissances relationnelles détenues ne semblent néanmoins pas être le seul élément permettant une application efficace du raisonnement analogique. En effet, la mémoire de travail et l'inhibition détiennent également un rôle non négligeable dans le développement du raisonnement analogique. Waltz, Lau, Grewal et Holyoak [6] observent les performances de participants à une tâche de raisonnement analogique utilisant des situations de crossmapping (dans lesquelles les similarités perceptuelles et relationnelles sont discordantes) associée ou non à une épreuve de rétention de chiffres. Les participants obtiennent de moins bonnes performances lorsque le fonctionnement de leur boucle phonologique est occupé par cette dernière tâche : ils choisissent en fait de mettre en correspondance les éléments partageant des caractéristiques physiques aux dépens des éléments occupant le même rôle relationnel. La mise en correspondance à partir d'éléments relationnels requerrait donc des ressources de mémoire de travail auditivo-verbale, contrairement à la mise en correspondance à partir d'éléments perceptuels.

L'inhibition, quant à elle, permet de se défaire des traits saillants et de se concentrer sur le traitement des relations. Morrison et ses collaborateurs [7] observent les performances de patients présentant une démence fronto-temporale avec des lésions prédominant au niveau du lobe frontal : ceux-ci choisissent préférentiellement d'utiliser les similarités perceptuelles plutôt que relationnelles dans des tâches analogiques qui requièrent l'inhibition des éléments perceptuels au profit des relations en présence. De plus, la modélisation statistique du raisonnement relationnel par le modèle LISA [3] révèle que l'inhibition permet de se focaliser sur les similarités relationnelles au détriment des similarités perceptuelles.

Enfin, la connaissance relationnelle et les capacités de mémoire de travail interagissent lors de la résolution d'analogies. En effet, l'accroissement des connaissances relationnelles relatives à une tâche permet de réduire la quantité de ressources cognitives nécessaires pour la 
traiter. Plus les connaissances relatives à un domaine sont importantes, plus les demandes en mémoire de travail sont réduites pour la résolution d'une tâche analogique [3].

\section{Raisonnement analogique et langage}

Le raisonnement analogique entretient un lien étroit avec le langage. Tout d'abord, il semble que l'utilisation du langage facilite le raisonnement analogique dans une tâche nonverbale. En effet, Son, Smith, Goldstone et Leslie [8] montrent que l'utilisation d'un mot évoquant la relation entretenue par les éléments d'une situation (partage pour un pattern croix-pingouin-croix) permet aux enfants de résoudre plus facilement une tâche de raisonnement analogique. L'effet de ce type de mot est supérieur à celui de non-mots nonreliés (koli pour croix-pingouin-croix), à celui de non-mots de structure similaire à celle de l'item (koliko pour cette même structure $\mathrm{ABA}$ ) et à celui de mots non-reliés (bouillant). Le langage détient donc un rôle important pour la découverte de la similarité relationnelle et le raisonnement analogique.

Inversement, le raisonnement analogique joue également un rôle primordial dans l'acquisition du langage. Il permet en effet de comprendre le sens d'un mot nouveau [9] mais aussi d'acquérir la grammaire de la langue. En effet, l'alignement des structures perçues permet à l'enfant de généraliser les constructions d'un verbe à l'autre et de détecter les régularités de la langue [9]. Ainsi, des enfants peuvent apprendre à reconnaître des régularités au sein de stimuli semblables aux stimuli langagiers. Marcus, Vijayan, Bandi Rao et Vishton [10], par exemple, ont observé que des bébés de 7 mois étaient capables d'apprendre une grammaire artificielle selon un processus d'abstraction de règles en plus du traitement statistique de l'information.

\section{Connaissance relationnelle et langage}

Gentner et Rattermann [11] constatent que l'acquisition des termes relationnels tels que les verbes ou les adjectifs relationnels se fait après l'acquisition des noms propres ou communs. Ainsi, les enfants acquièrent dans un premier temps le nom des objets séparés de leur contexte puis ils développent leur stock de mots relationnels. Une explication possible est que les objets sont plus faciles à extraire du flux d'expériences que le sont les relations [11]. De plus, les auteurs notent que lorsque les termes relationnels sont entrés dans le vocabulaire des enfants, ceux-ci ont encore besoin de temps pour acquérir leur signification précise. Le développement du lexique relationnel semble donc être postérieur au développement du 
lexique des objets concrets, ce qui peut être mis en parallèle avec l'hypothèse de la transition du perceptuel au relationnel soutenue par Gentner [11].

La connaissance relationnelle est également centrale en ce qui concerne l'acquisition et l'utilisation du langage. En effet, l'apprentissage de verbes nécessite d'acquérir les différents éléments qui composent la représentation de la relation qu'ils désignent. De plus, le langage est récursif tout comme la connaissance relationnelle. Ainsi, la connaissance relationnelle permet de générer de nouvelles formes : par exemple, si je connais la représentation du verbe aimer et les deux éléments qui sont reliés par lui (celui qui aime et celui qui est aimé), je peux former un nombre illimité de propositions [12]. Selon Gentner et Rattermann [11], le processus de re-representation serait primordial pour cet apprentissage. En effet, pour établir une correspondance entre deux situations, il convient de former une représentation plus abstraite de la relation que celle que l'on possède. Ainsi, en mettant en correspondance des situations partiellement similaires, les enfants se créent des représentations plus abstraites qui permettent d'en extraire les points communs. A chaque mise en correspondance fructueuse, la représentation devient plus abstraite et plus uniforme, favorisant alors la probabilité pour deux autres situations d'être mises en correspondance, et ainsi de suite.

\section{Dysphasie et raisonnement analogique}

La dysphasie est un trouble sévère et persistant du développement du langage oral [13]. Les critères diagnostiques d'inclusion, que sont les performances langagières inférieures à celles attendues au vu de l'âge de l'enfant, présentent autant d'importance dans la définition de la dysphasie que les critères d'exclusion. En effet, celle-ci atteint des enfants ayant des difficultés à acquérir le langage oral malgré une intelligence non-verbale dans la moyenne, une bonne audition, une absence de dysfonctionnement neurologique, un fonctionnement oromoteur normal et un environnement propice à l'apprentissage du langage oral [14]. La dysphasie se manifeste dans les différents domaines langagiers que sont notamment la phonologie, le lexique et la morphosyntaxe (voir pour une revue détaillée, Leonard, 2014).

Le premier signe de la dysphasie est souvent l'apparition tardive des premiers mots. Celle-ci est en effet régulièrement retardée d'environ un an [15]. De même, l'explosion lexicale que l'on observe habituellement vers 2 ans est souvent absente [16]. Les premières combinaisons de mots apparaissent également plus tard chez les enfants présentant une dysphasie (par après, enfants dysphasiques) : alors que les enfants tout-venant forment leurs 
premières combinaisons vers 1 an et demi, elles peuvent n'apparaitre que vers 3 ans chez les enfants dysphasiques [16]. Par la suite, ces enfants présentent un stock de vocabulaire plus pauvre que leurs pairs, ainsi qu'une moins bonne organisation lexico-sémantique. Ils peuvent aussi présenter des difficultés d'apprentissage de nouveaux mots ou d'accès lexical [17]. L'utilisation de la morphosyntaxe, et en particulier des morphèmes grammaticaux constitue également une difficulté majeure pour les enfants dysphasiques. Ceux-ci ont par exemple plus de difficultés à utiliser l'auxiliaire $a$ au passé composé par rapport à la préposition à [18].

Les enfants dysphasiques détiennent une intelligence non-verbale dans la norme et peuvent réaliser une tâche comme les matrices de Raven sans difficulté [19]. Cependant, lorsque des tâches de raisonnement analogique fin sont créées, les enfants dysphasiques sont mis en difficultés. Leroy, Parisse et Maillart [20] ont en effet montré que les enfants dysphasiques obtiennent des performances inférieures aux enfants contrôles de même âge chronologique dans une tâche analogique non langagière. De plus, les enfants dysphasiques sont davantage aidés par le fait que les items soient similaires au sein d'une même séquence. Ils sont donc mis en difficulté quand il s'agit de créer des analogies, c'est-à-dire de découvrir la similarité relationnelle existant entre deux situations grâce à un alignement de leur structure.

Ces difficultés se retrouvent dans l'acquisition du langage et plus particulièrement dans l'acquisition de la morphosyntaxe : en effet, l'enfant doit abstraire des constructions à partir des différentes formes linguistiques qu'il perçoit. Ainsi, il doit pouvoir comparer des items de type il voit un oiseau et il voit un bateau pour en arriver au schéma il voit un X. C'est donc grâce au raisonnement analogique que l'enfant peut découvrir la structure commune abstraite existant entre différentes formes. Les enfants dysphasiques, présentant un déficit dans ces capacités, sont plus lents et ont plus de difficultés à découvrir cette structure et à abstraire des schémas de construction [20]. Ils sont donc plus dépendants des formes lexicalisées et moins productifs. En outre, ils ont besoin que les différentes formes présentées soient davantage similaires sur le plan perceptuel pour pouvoir en dégager la similarité relationnelle [20].

Les enfants dysphasiques semblent présenter des difficultés liées au raisonnement analogique par rapport à leurs pairs de même âge. Au vu du lien existant entre le raisonnement analogique et le langage, ainsi qu'entre le raisonnement analogique et la mémoire de travail, il parait pertinent de se demander si les difficultés observées sont toujours 
présentes lorsque ces deux habiletés sont contrôlées. Cet article cible donc les questions suivantes : dans une tâche de raisonnement analogique,

1) les enfants dysphasiques sont-ils capables d'identifier des relations présentes entre deux éléments de façon similaire à leurs pairs appariés par niveau langagier ou par niveau de mémoire de travail ?

2) les enfants dysphasiques sont-ils davantage dépendants de la similarité perceptuelle entre les éléments que leurs pairs appariés par niveau langagier ou par niveau de mémoire de travail ?

\section{METHODOLOGIE}

\section{Participants}

Septante-six participants au total ont été évalués pour cette étude. Vingt-quatre enfants dysphasiques francophones ont été testés et appariés soit selon leur âge linguistique, soit selon une mesure de mémoire de travail, soit les deux. Onze de ces enfants ont également été appariés en âge chronologique afin de vérifier notre hypothèse de départ soutenant que le raisonnement analogique des enfants dysphasiques est altéré par rapport à leurs pairs de même âge. Ces participants ont été recrutés au sein de deux écoles spécialisées de la région liégeoise, en Belgique francophone. Chacun avait reçu un diagnostic de dysphasie au préalable. Les enfants bilingues ou ceux porteurs d'un trouble de la vision, de l'audition ou des habiletés oro-motrices ont été exclus. A noter également que les enfants dysphasiques sélectionnés détenaient tous un QI non verbal supérieur à $-1.5 \sigma$.

Cinquante-deux enfants contrôles ont été recrutés en milieu scolaire. Dix enfants ont participé au pré-test de la tâche expérimentale, et quarante-deux ont pris part à l'étude en ellemême. Deux enfants n'ont pas été repris en raison de performances particulièrement faibles lors de la passation de la tâche qui relevaient, soit d'une incompréhension des consignes, soit d'un désinvestissement de la situation de testing. Vingt enfants forment le groupe contrôle apparié en âge linguistique et vingt le groupe contrôle apparié en mémoire de travail. Onze de ces enfants ont aussi participé à l'appariement en âge chronologique. Les enfants bilingues et ceux présentant un trouble du langage oral autre qu'un trouble articulatoire isolé n'ont pas été sélectionnés pour la constitution du groupe contrôle. Les caractéristiques des groupes se retrouvent dans le tableau 1 en fonction des appariements.

\section{Matériel}


Pour apparier les enfants selon leur âge linguistique, la forme A du test Echelle de Vocabulaire en Images Peabody [21] a été utilisée. Cette épreuve permet d'évaluer le stock lexical en réception d'enfants et adolescents de 2 ans et demi à 18 ans. Pour l'appariement en mémoire de travail, l'empan de chiffres envers de la Wechsler Intelligence Scale for Children, quatrième édition [22] a été administré. Cette tâche permet d'évaluer les capacités de mémoire de travail en modalité auditivo-verbale. Elle se compose de séries de chiffres de longueur croissante, l'empan correspondant au nombre de chiffres rappelés au dernier item réussi.

Pour évaluer le raisonnement analogique des participants, nous avons créé une tâche inspirée de celle utilisée dans l'étude de Markman et Gentner [23]. Les participants ont pour tâche d'associer un élément pointé par une flèche dans une première image à l'élément qui détient le même rôle relationnel dans une deuxième image. Les différents items sont répartis dans trois conditions distinctes, un exemple étant repris dans la figure 1 pour chacune d'elles. Dans la première condition, dite facilitatrice, les éléments ayant le même rôle relationnel dans les deux scènes sont également perceptuellement similaires. La deuxième condition, dite neutre, comporte des items dans lesquels aucune similarité perceptuelle n'est présente d'une scène à l'autre. Enfin, dans la troisième condition, dite interférente, les informations perceptuelles et relationnelles sont en contradiction : les éléments perceptuellement similaires dans les deux scènes ne présentent pas de similarité relationnelle. La tâche a été conçue de manière à être accessible à des enfants d'âge scolaire. Aucun élément superflu n'a été ajouté dans les scènes afin d'éviter une surcharge cognitive liée au traitement d'informations distractrices.

Un pré-test de la tâche a été réalisé sur un échantillon de dix enfants pour s'assurer que les variations dans la réalisation des items (dessins en couleurs ou en noir et blanc, dessinateurs, placement des éléments sur le dessin) n'amenaient pas de différence de résultat. Le pré-test a également permis d'éliminer l'item le moins bien réussi de la condition neutre et de la condition interférente. Les exemples et la consigne ont aussi été fixés grâce au pré-test. La consigne est ainsi énoncée en s'appuyant sur l'item saluer de la condition neutre, pour lequel nous indiquons à l'enfant que deux personnages font la même chose et qu'ils vont donc ensemble. Nous soulignons ensuite l'importance de bien regarder les deux scènes et de pointer l'élément en bas qui va bien avec celui pointé par la flèche en haut. Un exemple tiré de la condition facilitatrice puis de la condition interférente est également présenté à l'enfant.

\section{Procédure}


Les enfants ont été évalués à l'école ou à la maison dans une pièce calme et isolée. Pour les participants contrôles, la passation a été réalisée en une seule séance qui débutait par la tâche expérimentale. Suivait ensuite l'administration de l'EVIP [21] et/ou de l'empan de chiffres endroit puis envers [22]. Les enfants dysphasiques ont été rencontrés à deux reprises : lors d'une première séance, l'EVIP [21] et la tâche d'empans [22] étaient administrés et lors d'une deuxième, la tâche expérimentale leur était proposée. Au total, la passation durait entre trente et quarante minutes.

La tâche expérimentale créée contient trois exemples et trente items répartis dans trois conditions distinctes. La passation est effectuée sur ordinateur à l'aide du logiciel E-Prime 2.0 [24]. Les items sont présentés dans un ordre aléatoire pour neutraliser les effets de fatigue ou les biais liés à un ordre fixe. Aucune verbalisation et aucun feedback correctif n'est produit pendant la réalisation de la tâche en elle-même pour éviter l'augmentation des temps de réponse et un possible effet d'apprentissage. L'enfant doit uniquement pointer les deux personnages jouant le même rôle relationnel. Le passage à l'item suivant est effectué par l'examinateur aussitôt que l'enfant a produit sa réponse. L'administration des tâches d'appariement s'est effectuée dans le respect des conditions de standardisation stipulées par les manuels, le participant obtenant un point par réponse correcte.

\section{RESULTATS}

Pour les différentes analyses, les scores de réussite ont été mis au carré pour obtenir une distribution normale des données. Des ANOVA à mesures répétées ont été utilisées et les résultats ont été interprétés grâce à un $\alpha$ de .05 . Lorsque l'hypothèse de sphéricité n'était pas rencontrée, le correctif de Greenhouse et Geisser était utilisé.

\section{Appariement en âge chronologique}

Une analyse de variance a été conduite sur les scores de réussite, avec le groupe (enfants dysphasiques vs. enfants contrôles) en variable indépendante inter-sujets et la condition (facilitatrice, neutre et interférente) en variable indépendante intra-sujets. Les résultats sont repris dans la figure 2. Nous obtenons un effet d'interaction, la réussite des différentes conditions variant en fonction du groupe, $F(2,20)=5.215, p<.05$. Les scores des deux groupes ne diffèrent en fait ni pour la condition facilitatrice $(p=.99)$, ni pour la condition neutre $(p=.99)$. On observe par contre une différence de résultats pour la condition interférente $(p<.01)$, les enfants contrôles ayant de meilleures performances que ceux présentant une dysphasie. De même, les enfants dysphasiques réussissent mieux la condition 
facilitatrice que la condition interférente $(p<.05)$ alors que les résultats des enfants contrôles sont similaires pour ces deux conditions ( $p=.97)$. L'effet du groupe est également significatif, $F(1,10)=8.251, p<.05$, les participants contrôles ayant de meilleures performances que les participants dysphasiques. Nous n'obtenons par contre pas de différence entre les conditions quel que soit le groupe d'appartenance des participants, $F(2,20)=2.678, p>.05$.

\section{Appariement en âge linguistique}

Les résultats se trouvent dans le tableau 2. Les deux groupes appariés en âge linguistique obtiennent des performances similaires, $F(1,19)=0.001, p>.05$. Par contre, les trois conditions ne sont pas réussies de la même manière, $F(1.35,25.70)=5.293, p<.05$. Les comparaisons post hoc de Tukey indiquent que la réussite de la condition facilitatrice diffère de celle des conditions neutre $(p<.05)$ et interférente $(p<.05)$, ces dernières étant moins bien réussies par l'ensemble des enfants. Aucune différence n'apparaît par contre entre ces deux dernières conditions $(p=.92)$. Enfin, aucun effet d'interaction n'est présent, $F(1.44,27.32)=$ $1.016, p>.05$ : la réussite des différentes conditions ne diffère donc pas en fonction du groupe des participants.

\section{Appariement en mémoire de travail}

Les données sont reprises dans le tableau 3. Les deux groupes appariés en mémoire de travail obtiennent des résultats similaires, $F(1,19)=4.004, p>.05$. Par contre, les scores diffèrent selon la condition, $F(1.44,27.42)=9.943, p<.01$. La condition facilitatrice est significativement mieux réussie que les conditions neutre $(p<.01)$ et interférente $(p<.01)$. Aucune différence n'apparaît par contre entre ces deux dernières conditions $(p=.89)$. Ces résultats rejoignent ceux observés sur les données de l'appariement en âge linguistique. Enfin, la réussite selon la condition est similaire entre les deux groupes puisqu'aucun effet d'interaction n'est présent, $F(1.55,29.50)=0.958, p>.05$.

\section{DISCUSSION}

L'objectif principal de cette étude était d'évaluer les capacités d'identification de relations d'enfants dysphasiques et de les comparer à celles d'enfants appariés par niveau langagier ou par niveau de mémoire de travail. La dépendance à la similarité perceptuelle présente entre les éléments devait également être analysée.

\section{Développement du raisonnement analogique dans la dysphasie}


Les enfants dysphasiques semblent détenir des connaissances relationnelles comparables à celles de pairs de même âge linguistique ou de mêmes capacités de mémoire de travail, mais aussi à celles de pairs de même âge chronologique. Aucune différence n'apparait en effet entre les groupes lorsqu'aucun élément perceptuel n'est commun entre les deux scènes. Ceci est en accord avec certaines données de la littérature qui montrent des performances similaires entre des enfants ayant des troubles langagiers et leurs pairs de même âge. Ainsi, Kamhi, Gentry, Mauer et Gholson [25] n'observent aucune différence de groupe à une tâche de résolution de problème lorsque celui-ci est présenté oralement et accompagné de démonstrations physiques. Néanmoins, d'autres études n'obtiennent pas les mêmes résultats. En effet, Masterson, Evans et Aloia [26] constatent que des enfants ayant des troubles langagiers ont des performances inférieures à des pairs de même âge mental légèrement plus jeunes. A noter que leur étude impliquait des analogies verbales. Leroy et ses collaborateurs [20] montrent également que des enfants dysphasiques obtiennent des performances inférieures à des pairs de même âge chronologique à une tâche analogique non-verbale. Il est cependant intéressant de noter que dans cette étude, aucune différence n'apparait entre les groupes lorsque deux caractéristiques perceptuelles sont partagées par les formes géométriques d'une même séquence. Cette dernière donnée est quant à elle en accord avec nos résultats puisqu'aucune différence n'apparait entre les enfants dysphasiques et leurs pairs de même âge pour la condition facilitatrice.

Cependant, nous avons également constaté l'apparition de difficultés chez les enfants dysphasiques par rapport à leurs pairs de même âge chronologique lorsqu'une information perceptuelle distractrice est introduite. Bien que peu d'études aient évalué cet effet, Leroy et ses collaborateurs [20] ont démontré que les enfants dysphasiques semblaient davantage dépendants à la similarité perceptuelle que leurs pairs de même âge. En effet, tous les participants de l'étude voyaient leurs performances réduites avec la diminution de la similarité perceptuelle présente entre les formes d'une même séquence. Néanmoins, cette diminution était plus marquée chez les enfants dysphasiques que chez leurs pairs de même âge chronologique. Or, cette dépendance à la similarité perceptuelle et les difficultés liées à la réalisation de notre condition interférente semblent pouvoir être expliquées par les troubles langagiers et de mémoire de travail présents dans la dysphasie. En effet, lorsque ces capacités sont contrôlées, aucune différence n'apparait entre les enfants dysphasiques et les enfants contrôles, et ce y compris pour la condition interférente. Masterson et ses collaborateurs [26] obtiennent le même type de résultats puisque, dans leur étude, les enfants ayant des troubles langagiers et leurs pairs de même âge linguistique ont des performances similaires à une tâche 
impliquant des analogies verbales. Nous avons déjà vu que les habiletés langagières et de mémoire de travail permettaient de favoriser la prise en compte du relationnel et l'inhibition des indices perceptuels distracteurs [6][7]. Les capacités langagières et de mémoire de travail [27] étant altérées chez les enfants dysphasiques, ceux-ci pourraient être mis en difficultés par rapport à leurs pairs de même âge chronologique quand un élément perceptuel distracteur est introduit au sein d'une tâche analogique.

Néanmoins, il est également possible que des déficits plus subtils de connaissance relationnelle puissent mettre les enfants dysphasiques en difficulté lorsqu'une tâche analogique se complexifie. En effet, la connaissance relationnelle interagit avec les capacités de mémoire de travail : ainsi, des connaissances relationnelles plus développées permettraient de réduire les demandes en mémoire de travail nécessaires à la résolution d'une tâche analogique [28]. De même, le langage permet d'améliorer les connaissances relationnelles et les performances analogiques en situation de conflit, lorsque les fonctions exécutives doivent intervenir [28]. Ainsi, une connaissance relationnelle approfondie permet d'encoder les informations relationnelles plus efficacement. Associée à des termes relationnels, l'attention sera plus facilement focalisée sur les relations et les indices perceptuels non pertinents pourront être aisément inhibés. La connaissance relationnelle n'est donc pas une composante du raisonnement analogique qui se définit selon un codage binaire présente ou absente. Elle peut influencer le raisonnement analogique de manière plus subtile, en fonction de la robustesse de ses représentations [28].

\section{Influence de l'information perceptuelle sur la résolution d'une tâche analogique}

$\mathrm{Au}$ vu de nos résultats, la condition facilitatrice semble mieux réussie que les deux autres, du moins jusqu'à un certain âge. La congruence entre informations perceptuelles et relationnelles semble donc être bénéfique pour les enfants dysphasiques et pour les enfants tout-venant les plus jeunes. Cet effet bénéfique n'est en effet pas retrouvé chez les enfants contrôles appariés en âge chronologique, ceux-ci étant les plus âgés de notre groupe contrôle. Ces données vont à l'encontre des résultats de Goswami et Brown [5], qui soutiennent que les enfants n'ont pas besoin d'indices perceptuels pour résoudre une tâche relationnelle. En effet, dans leur étude, des enfants de seulement 3 ans peuvent résoudre une tâche analogique pour peu qu'ils connaissent les relations en présence. De plus, l'ajout de similarité perceptuelle n'influençait pas les résultats. Celle-ci n'est donc utile que si les relations sont complexes et non-familières aux participants [5]. 
Or, les relations utilisées dans notre tâche sont supposées être familières aux enfants recrutés et correspondent à des verbes fréquents pour leur âge. Il ne semble donc pas que des difficultés majeures de connaissance des relations aient pu influencer ces résultats. Ainsi, nos résultats peuvent être analysés à la lumière des résultats d'une autre étude avec laquelle ils s'avèrent plus compatibles. En effet, des auteurs [20] obtiennent des données assez différentes de celles de Goswami et Brown [5] quant à l'influence de la similarité perceptuelle sur la résolution d'une tâche analogique. Dans leur étude, les participants devaient compléter une séquence de formes géométriques selon un modèle présenté dans deux séquences antérieures. Les résultats des participants étaient meilleurs si les différentes séquences étaient similaires d'un point de vue perceptuel (c'est-à-dire de même couleur). De plus, leurs performances étaient meilleures si les éléments d'une même séquence partageaient deux traits communs (soit la couleur et la forme), par rapport aux séquences dans lesquelles un trait était commun ou dans lesquelles aucun trait commun n'était présent. Cette étude apporte donc des éléments dans le sens d'une facilitation de la résolution d'une tâche analogique par la présence de similarité perceptuelle, qu'elle soit inter- ou intra-séquence [20]. Simms [28] propose une explication de cette facilitation. En effet, elle observe que l'attention des enfants, contrairement à celle des adultes, est dirigée vers les objets et les indices perceptuels avant de se tourner vers les relations. Dans le cas où la similarité perceptuelle correspond à la similarité relationnelle, l'attention portée dans un premier temps sur les indices perceptuels mènera directement à la prise en compte des indices relationnels [28].

Un autre effet retrouvé dans la littérature est celui lié à l'information perceptuelle distractrice. Plusieurs auteurs obtiennent en effet une baisse des résultats lorsque de telles informations sont introduites. Thibaut, French et Vezneva [29] obtiennent une différence significative à une tâche relationnelle entre une condition avec et une condition sans distracteur perceptuel, cette dernière étant mieux réussie. Richland, Morrison et Holyoak [30] mettent eux aussi en évidence l'influence négative d'un distracteur perceptuel, cette influence disparaissant chez les participants de plus de 9 ans. Ces éléments ne se retrouvent pas dans nos données. Ceci peut être expliqué par des imperfections dans la réalisation des items, notamment certains de la condition neutre qui semblent plus difficiles à comprendre que ceux de la condition interférente.

\section{Perspectives}

Certaines limites ont pu être relevées au cours de la réalisation de notre étude. Tout d'abord, l'échantillon est relativement restreint. Cette étude met en évidence l'intérêt de 
disposer de différents groupes contrôles. A l'avenir, il serait utile de diversifier les mesures utilisées (par exemple, avoir plusieurs mesures de mémoire de travail) pour mieux cerner les compétences des enfants et leurs profils.

Malgré la réalisation d'un pré-test pour valider les items de la tâche expérimentale, certains items ont semblé mettre davantage les enfants en difficulté par rapport à d'autres, en particulier dans la condition neutre. La tâche créée ici permet d'évaluer une relative préservation de la connaissance relationnelle mais ne permet pas de juger avec précision de la qualité de cette connaissance. L'introduction d'une variable prenant en compte la complexité relationnelle pourrait permettre d'analyser cette qualité, en plus de celle permettant d'évaluer la résistance à la présence de distracteurs perceptuels.

\section{CONCLUSION}

Les enfants dysphasiques disposent de capacités de raisonnement analogique similaires à celles de leurs pairs de même âge chronologique dans des situations simples. En revanche, ils sont mis en difficulté lorsque des informations perceptuelles distractrices sont introduites. Ces difficultés disparaissant lorsque les habiletés langagières et de mémoire de travail sont contrôlées, on peut supposer que leurs difficultés de raisonnement analogique seraient liées aux déficits de ces deux habiletés présents dans la dysphasie. Néanmoins, une altération plus subtile des connaissances relationnelles n'est pas à exclure, bien que celles-ci semblent préservées a priori. Dans ce cadre, une tâche de complexité relationnelle variée ou encore une tâche d'apprentissage relationnel pourrait être proposée. De même, l'influence des capacités de raisonnement analogique sur les troubles langagiers présents dans la dysphasie devrait être précisée.

\section{REFERENCES}

[1] Gentner D, Colhoun J. Analogical processes in human thinking and learning. In : Glatzeder B, Goel V, Müller A, eds. Towards a theory of thinking. Berlin : Springer Berlin Heidelberg, $2010: 35-48$.

[2] Goswami U. Analogical reasoning: What develops? A review of research and theory. Child Development $1991 ; 62: 1-22$.

[3] Morrison RG, Doumas LA, Richland LE. A computational account of children's analogical reasoning: Balancing inhibitory control in working memory and relational representation. Developmental Science 2011 ; 14 : 516-529. 
[4] Gentner D. Metaphor as structure mapping: The relational shift. Child Development 1988 ; 59 : 47-59.

[5] Goswami U, Brown AL. Melting chocolate and melting snowmen: Analogical reasoning and causal relations. Cognition $1990 ; 35: 69-95$.

[6] Waltz JA, Lau A, Grewal SK, et al. The role of working memory in analogical mapping. Memory \& Cognition 2000 ; 28 : 1205-1212.

[7] Morrison RG, Krawczyk DC, Holyoak KJ, et al. A neurocomputational model of analogical reasoning and its breakdown in frontotemporal lobar degeneration. Journal of Cognitive Neuroscience 2004 ; 16 : 260-271.

[8] Son JY, Smith LB, Goldstone RL, et al. The importance of being interpreted: Grounded words and children's relational reasoning. Frontiers in Psychology 2012 ; 3 : 45 57.

[9] Gentner D, Namy LL. Analogical processes in language learning. Current Directions in Psychological Science 2006 ; 15 : 297-301.

[10] Marcus GF, Vijayan S, Bandi Rao S, et al. Rule-learning by seven-month-old infants. Science $1999 ; 283: 77-80$.

[11] Gentner D, Rattermann MJ. Language and the career of similarity. In : Gelman SA, Byrnes JP, eds. Perspectives on language and thought: Interrelations in development. New York : Cambridge University Press, 1991 : 225-277.

[12] Halford GS, Wilson WH, Phillips S. Relational knowledge: The foundation of higher cognition. Trends in Cognitive Sciences 2010 ; 14 : 497-505.

[13] Soares-Boucaud I, Labruyère N, Jery S, et al. Dysphasies développementales ou troubles spécifiques du développement du langage. EMC Pédopsychiatrie 2009 ; 37-201-E15.

[14] Leonard LB. Children with specific language impairment. Cambridge (USA) : The MIT Press, 2014.

[15] Trauner D, Wulfeck B, Tallal P, et al. Neurological and MRI profiles of children with developmental language impairment. Developmental Medicine \& Child Neurology $2000 ; 42: 470-475$.

[16] Leclercq A-L, Leroy S. Introduction générale à la dysphasie : Caractéristiques linguistiques et approches théoriques. In : Maillart C, Schelstraete M-A, eds. Les dysphasies : De l'évaluation à la réeducation. Issy-les-Moulineaux : Elsevier Masson, 2012 : 5-33.

[17] Sheng L, McGregor KK. Lexical-semantic organization in children with specific language impairment. Journal of Speech, Language, and Hearing Research 2010 ; 53 : 146159. 
[18] Paradis J, Crago M. Tense and temporality: A comparison between children learning a second language and children with SLI. Journal of Speech, Language \& Hearing Research $2000 ; 43: 834-847$.

[19] Van Daal J, Verhoeven L, van Balkom H. Cognitive predictors of language development in children with specific language impairment (SLI). International Journal of Language \& Communication Disorders 2009 ; 44 : 639-655.

[20] Leroy S, Parisse C, Maillart C. Analogical reasoning in children with specific language impairment. Clinical Linguistics \& Phonetics 2012 ; 26 : 380-395.

[21] Dunn LM, Theriault-Whalen CM, Dunn LM. Echelle de vocabulaire en images peabody. Toronto (Canada) : Psychan, 1993.

[22] Wechsler D. WISC IV - Wechsler intelligence scale for children - fourth edition. San Antonio (USA) : The Psychological Corporation, 2005.

[23] Markman AB, Gentner D. Structural alignment during similarity comparisons. Cognitive Psychology 1993 ; 25 : 431-467.

[24] Psychology Software Tools, Inc. E-Prime 2.0. Retrieved from http://www.pstnet.com, 2012.

[25] Kamhi AG, Gentry B, Mauer D, Gholson B. Analogical learning and transfer in language-impaired children. Journal of Speech \& Hearing Disorders 1990 ; 55 : 140-148.

[26] Masterson JJ, Evans LH, Aloia M. Verbal analogical reasoning in children with language-learning disabilities. Journal Of Speech \& Hearing Research 1993 ; 36 : 76-82.

[27] Gathercole SE, Baddeley AD. Phonological memory deficits in language disordered children: Is there a causal connection? Journal of Memory and Language $1990 ; 29$ :336-360.

[28] Simms NK. Overcoming the object bias in analogical development: Encoding time, allocation of attention, and relational knowledge. Dissertation Abstracts International: Section B: The Sciences and Engineering 2014 ; 75(1) : no pagination specified.

[29] Thibaut JP, French R, Vezneva M. The development of analogy making in children: Cognitive load and executive functions. Journal of Experimental Child Psychology $2010 ; 106: 1-19$.

[30] Richland LE, Morrison RG, Holyoak KJ. Children's development of analogical reasoning: Insights from scene analogy problems. Journal of Experimental Child Psychology 2006 ; $94: 249-273$. 


\begin{tabular}{|c|c|c|c|}
\hline \multicolumn{4}{|c|}{ APPARIEMENT EN AGE CHRONOLOGIQUE } \\
\hline & Dysphasique & Contrôle & Statistiques \\
\hline Sexe & 8 garçons ; 3 filles & 7 garçons ; 4 filles & $\begin{array}{c}\text { Chi }{ }^{2} \text { exact de Fisher } \\
p>.05\end{array}$ \\
\hline $\begin{array}{l}\text { Age chronologique } \\
\text { moyen (Ecart-type) }\end{array}$ & $8.58(0.87)$ & $8.54(0.85)$ & $\begin{array}{c}t \text { de Student pour } \\
\text { échantillons indépendants } \\
t(20)=-0.121, p>.05\end{array}$ \\
\hline \multicolumn{4}{|c|}{ APPARIEMENT EN AGE LINGUISTIQUE } \\
\hline Sexe & 14 garçons ; 6 filles & 11 garçons ; 9 filles & $\begin{array}{c}\text { Chi }{ }^{2} \text { exact de Fisher } \\
p>.05\end{array}$ \\
\hline $\begin{array}{l}\text { Age chronologique } \\
\text { moyen (Ecart-type) }\end{array}$ & $10.18(1.76)$ & $7.27(1.48)$ & $\begin{array}{c}t d e \text { Student pour } \\
\text { échantillons indépendants } \\
t(38)=-5.664, p<.05\end{array}$ \\
\hline $\begin{array}{l}\text { Age linguistique } \\
\text { moyen (Ecart-type) } \\
\text { EVIP }\end{array}$ & $93.65(25.00)$ & $92.45(25.82)$ & $\begin{array}{c}t \text { de Student pour } \\
\text { échantillons indépendants } \\
t(38)=-0.149, p>.05\end{array}$ \\
\hline \multicolumn{4}{|c|}{ APPARIEMENT EN MEMOIRE DE TRAVAIL } \\
\hline Sexe & 15 garçons ; 5 filles & 11 garçons ; 9 filles & $\begin{array}{c}\text { Chi }{ }^{2} \text { exact de Fisher } \\
p>.05\end{array}$ \\
\hline $\begin{array}{l}\text { Age chronologique } \\
\text { moyen (Ecart-type) }\end{array}$ & $10.39(1.98)$ & $7.19(1.81)$ & $\begin{array}{c}t \text { de Student pour } \\
\text { échantillons indépendants } \\
t(38)=-5.332, p<.05\end{array}$ \\
\hline $\begin{array}{l}\text { Empan de chiffres } \\
\text { envers moyen (Ecart- } \\
\text { type) WISC IV }\end{array}$ & $2.55(0.89)$ & $2.55(0.89)$ & $\begin{array}{c}C h i^{2} \text { de Pearson } \\
\text { Chi }^{2}(3)=0.000, p>.05\end{array}$ \\
\hline
\end{tabular}

TABLEAU 1 - Caractéristiques des groupes en fonction des appariements 


\begin{tabular}{|l|c|c|c|}
\hline \multicolumn{4}{|c|}{ SCORES DE REUSSITE MOYENS (ECARTS-TYPES) SUR 100 } \\
\hline & Condition facilitatrice & Condition neutre & Condition interférente \\
\hline Contrôle & $90.80(13.45)$ & $80.20(17.20)$ & $82.90(28.72)$ \\
\hline Dysphasique & $95.35(10.12)$ & $82.55(14.53)$ & $76.40(31.35)$ \\
\hline
\end{tabular}

TABLEAU 2 - Résultats des groupes appariés en âge linguistique 


\begin{tabular}{|l|c|c|c|}
\hline \multicolumn{4}{|c|}{ SCORES DE REUSSITE MOYENS (ECARTS-TYPES) SUR 100 } \\
\hline & Condition facilitatrice & Condition neutre & Condition interférente \\
\hline Contrôle & $89.00(14.52)$ & $70.60(20.42)$ & $68.35(30.65)$ \\
\hline Dysphasique & $92.80(14.41)$ & $77.85(19.46)$ & $83.90(26.20)$ \\
\hline
\end{tabular}

TABLEAU 3 - Résultats des groupes appariés en mémoire de travail 


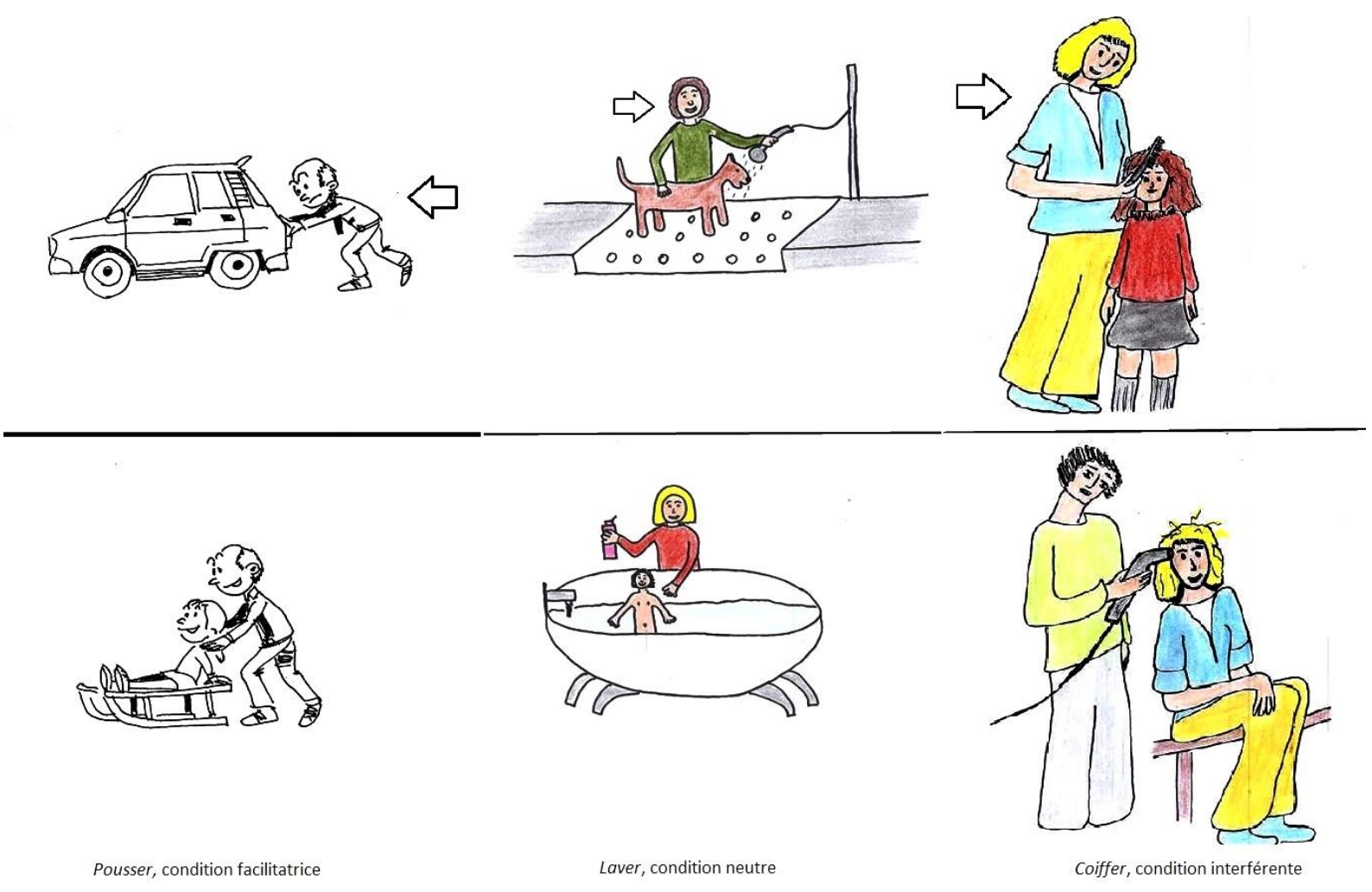

FIGURE 1 - Exemples d'items pour chaque condition 


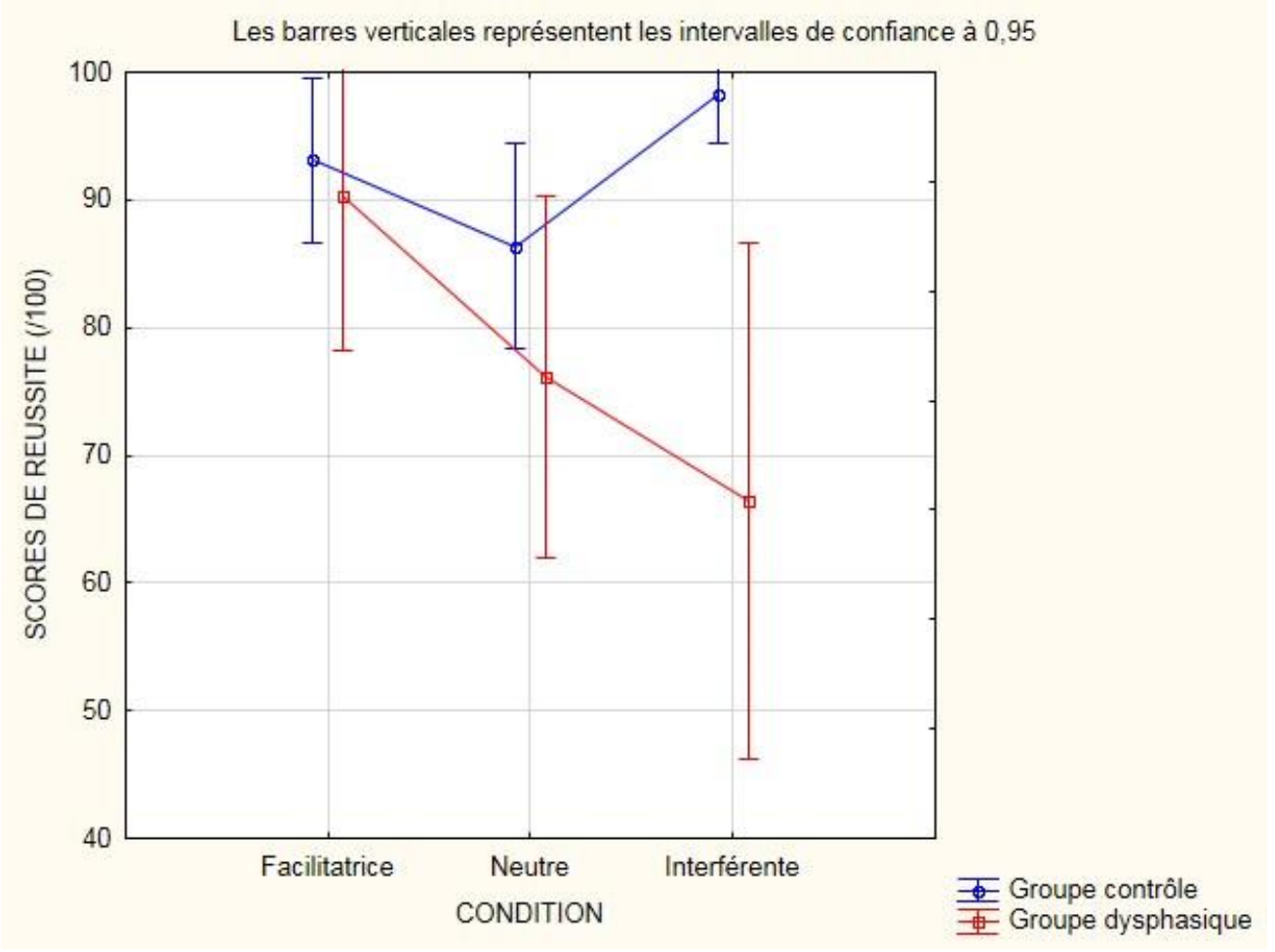

FIGURE 2 - Résultats des groupes appariés en âge chronologique 